\title{
Predicting numerically the large increases in extra pressure drop when boger fluids flow through axisymmetric contractions
}

\author{
H. R. Tamaddon-Jahromi ${ }^{1}$, M. F. Webster ${ }^{1^{*}}$, K. Walters ${ }^{2}$ \\ ${ }^{1}$ Institute of Non-Newtonian Fluid Mechanics, School of Engineering, Swansea University, Swansea, UK; m.f.webster@swan.ac.uk \\ ${ }^{2}$ Institute of Mathematics and Physics, University of Aberystwyth, Aberystwyth, UK
}

Received 10 October 2009; revised 3 November 2009; accepted 5 November 2009.

\begin{abstract}
Recent numerical studies on pressure-drops in contraction flows have introduced a variety of constitutive models to compare and contrast the competing influences of extensional viscosity, normal stress and shear-thinning. Early work on pressure-drops employed the constant viscosity Oldroyd-B and Upper Convected Maxwell (UCM) models to represent the behavior of so-called Boger fluids in axisymmetric contraction flows, in (unsuccessful) attempts to predict the very large enhancements that were observed experimentally. In more recent studies, other constitutive models have been employed to interpret observed behavior and some progress has been made, although finding a (respectable) model to describe observed contraction-flow behavior, even qualitatively, has been frustratingly difficult. With this in mind, the present study discusses the ability of a wellknown FENE type model (the so-called FENECR model) to describe observed behavior. For various reasons, an axisymmetric $(4: 1: 4)$ contraction/expansion geometry, with rounded corners, is singled out for special attention, and a new hybrid finite element/volume algorithm is utilized to conduct the modeling, which reflects an incremental pressure-correction time-stepping structure. New to this algorithmic formulation are techniques in time discretization, discrete treatment of pressure terms, and compatible stress/velocity-gradient representation. We shall argue that the current simulations for the FENE-CR model have resulted in a major improvement in the sort-for agreement between theory and experiment in this important bench-mark problem.
\end{abstract}

Keywords: Contraction Flows; Extra Pressure Drop;
Numerical Simulation; Extensional Viscosity; Normal Stress Differences

\section{INTRODUCTION}

In the early days of Computational Rheology, the socalled Upper-Convected Maxwell (UCM) and Oldroyd-B models were strongly favored. This was partly due to the fact that they were assumed to be the 'bottom-line' of acceptable mathematical simplicity, whilst also being able to mimic the complex rheometrical behavior for a class of dilute polymer solutions known as Boger fluids, which became popular in the late 1970s. (A Boger fluid has a reasonably constant shear viscosity, a high extensional viscosity as the extensional strain rate increases, and a high first normal stress difference, which has a quadratic dependence on shear rate at low to moderate shear rates, before becoming weaker than quadratic at higher shear rates.)

It is well known that simulations for the UCM and Oldroyd B models failed to predict the large increases in the so-called Couette correction (or equivalently the "extra pressure difference" (epd)) in axisymmetric contraction flows. Interestingly, the work of Debbaut and Crochet [1], Debbaut et al. [2] and Binding [3] already provided strong hints as to the likely cause of the inadequacies of these models in predicting the observed increases in excess pressure drop (epd) found in experimental work.

On the experimental side, Nigen and Walters [4] found significant differences in pressure-drop between Boger and Newtonian liquids with the same shear viscosity for axisymmetric contraction flow. (However, no distinction could be drawn between corresponding pressure drops for Newtonian and Boger fluids in planar configurations). Around the same time, Rothstein and McKinley [5] switched attention to axisymmetric contraction/expansion geometries of various contraction ratios (between two and eight), various degrees of re- 
ntrant corner curvature, and covering a large range of Deborah numbers. In this work, large Couette corrections were observed above that for a Newtonian fluid. These were independent of contraction ratio and rentrant corner curvature.

So, the increase in the Couette correction for Boger fluids flowing in various axisymmetric contractions can be very high. These experimental observations clearly presented theoretical (computational) rheologists with several significant challenges, some of which have already been resolved (see for example, Phillips and Williams [6]; Aboubacar et al. [7]; Walters and Webster [8]; Alves et al. [9]).

The present paper is a continuation of our previous works (Aguayo et al. [10]; Walters et al. [11-13], in predicting pressure-drops for Boger fluids in expansioncontraction flow. As a general conclusion from our earlier findings (particularly those in Walters et al. $[12,13]$ ), our numerical simulations confirmed earlier comments by Binding [3] and Debbaut and Crochet [1] that, whereas high extensional viscosity levels can give rise to large increases in the so-called epd, increasing normal-stress difference levels can have the opposite effect. Moreover, in Walters et al. [12,13], we attempted to show how generalizations of the so-called White-Metzner model (White and Metzner [14]) can help rheologists to understand the competing influence of the various rheometrical functions on important flow characteristics.

In this paper, a further numerical study of the $4: 1: 4$ expansion/contraction (adopted in Rothstein and McKinley [5], and Szabo et al. [15]) is made, and the effect on the excess pressure drop (epd) analyzed. In particular, we employ the FENE-type model proposed by Chilcott and Rallison [16], useful for its constant shear viscosity and bounded extensional characteristics. As in previous work, we attempt to relate the observed behavior of highlyelastic Boger fluids in complex flows to their rheometrical behavior.

\subsection{Rheometrical Functions}

In this communication, we shall be referring frequently to two important 'rheometrical' flows, namely steady simple shear flow and extensional flow. In the former, there is flow only in the $\mathrm{x}$ direction and this depends simply and linearly on the $y$ coordinate. i.e.

$$
\mathrm{v}_{\mathrm{x}}=\dot{\gamma} \mathrm{y}, \quad \mathrm{v}_{\mathrm{y}}=\mathrm{v}_{\mathrm{z}}=0
$$

where $\mathrm{v}_{\mathrm{i}}$ is the velocity vector and $\dot{\gamma}$ is the constant shear rate.

For a non-Newtonian elastic liquid, the stress tensor components $\sigma_{i k}$ can be conveniently written in the form:

$$
\begin{aligned}
& \sigma_{x y}=\sigma=\dot{\gamma} \eta(\dot{\gamma}) \\
& \sigma_{x x}-\sigma_{y y}=\mathrm{N}_{1}(\dot{\gamma}) \\
& \sigma_{y y}-\sigma_{z z}=\mathrm{N}_{2}(\dot{\gamma})
\end{aligned}
$$

where $\sigma$ is the shear stress, $\eta$ is the shear viscosity and $\mathrm{N}_{1}$ and $\mathrm{N}_{2}$ are the so-called first and second normal stress differences, respectively (see, for example, Barnes et al. [17]).

For a Newtonian fluid, the stress distribution simply involves one material constant-the coefficient of viscosity $\eta$ and the two normal stress differences are zero. In the case of a non-Newtonian elastic liquid, $\eta$ can now be a function of the shear rate, with so-called 'shear thinning' the most commonly observed behavior. Also, both normal stresses are of potential importance, particularly $\mathrm{N}_{1}$.

A typical Boger fluid would be a dilute (often a very dilute) solution of a high molecular weight polymer in a very viscous 'Newtonian' solvent. Usually, the shear viscosity is constant for such fluids; $\mathrm{N}_{1}$ is often higher than the shear stress $\sigma$, indicating that the fluid is in the "highly-elastic" category. $\mathrm{N}_{2}$ is invariably found to be much smaller than $\mathrm{N}_{1}$ and, in many (most) computational studies, $\mathrm{N}_{2}$ is taken to be zero.

At this point, it is important to stress that, from a continuum mechanics standpoint, the initial dependence of $\mathrm{N}_{1}$ on $\dot{\gamma}$ has to be quadratic and there is experimental evidence that this quadratic dependence can persist over a reasonable range of shear rates. However, there is also rheometrical evidence available that the dependence of $\mathrm{N}_{1}$ on $\dot{\gamma}$ can become weaker than quadratic as the shear rate increases further. This is sometimes accompanied by slight shear thinning. For example, in a comprehensive study entitled "A rheometrical study of Boger fluids", Jackson et al. [18] concluded "It will be seen that over a range of shear rates, $\sigma$ is a linear function of $\dot{\gamma}$ and $\mathrm{N}_{1}$ is a quadratic function of $\dot{\gamma}$, but that there is a departure from this second-order behavior at the high shear rates".

The second rheometrical flow of importance in the present study is that called 'uniaxial extensional flow', with a velocity field which can be expressed as

$$
\mathrm{v}_{\mathrm{x}}=\dot{\varepsilon} \mathrm{x}, \quad \mathrm{v}_{\mathrm{y}}=-\frac{\dot{\varepsilon} \mathrm{y}}{2}, \quad \mathrm{v}_{\mathrm{z}}=-\frac{\dot{\varepsilon} \mathrm{z}}{2}
$$

where $\dot{\varepsilon}$ is the so-called extensional strain rate. We can write the corresponding stress distribution in the form:

$$
\sigma_{x x}-\sigma_{y y}=\sigma_{x x}-\sigma_{z z}=\dot{\varepsilon} \eta_{E}(\dot{\varepsilon})
$$

where $\eta_{E}$ is the 'extensional viscosity'. For a Newtonian fluid, $\eta_{E}=3 \eta$, a result first obtained by Trouton over a hundred years ago (see, for example, Tanner and 
Walters [19]). For this reason, the ratio between the two viscosities is called the 'Trouton ratio $T_{R}$ ', and this clearly takes the value 3 for a Newtonian fluid. For a non-Newtonian elastic fluid, $\mathrm{T}_{\mathrm{R}}$ can be significantly higher than 3, with 'orders of magnitude' increases not uncommon.

From the above discussion and the relevant literature, we can associate the following rheometrical behavior with Boger fluids:

1) A reasonably constant shear viscosity $\eta$.

2) A potentially high extensional viscosity $\eta_{E}$ as the extensional strain rate increases.

3) A high first normal stress difference $N_{1}$, which has a quadratic shear rate dependence on $\dot{\gamma}$, at least for small to moderate shear rates.

4) A second normal stress difference $\mathrm{N}_{2}$ which is negative and at most one tenth of the magnitude of $\mathrm{N}_{1}$. It is often taken as zero in computational studies.

Clearly, any constitutive model that we use to describe Boger fluids has to satisfy (1)-(4), at least in a semiquantitative sense.

\section{THE CONTRACTION/EXPANSION PROBLEM}

In conventional contraction flow, liquids are forced through a contraction under a pressure gradient. At specific locations on the walls, upstream and downstream of the contraction, pressure measurements are made. These locations must be far enough away from the contraction for the flows to be deemed to be unaffected by the contraction, i.e. we may consider the flows to be 'fully developed' and 'Poiseuille like' at the pressure-measurement stations.

In contraction flows, there is often significant interest in the kinematics of the flow structure, particularly the vortices which provide computational rheologists with significant challenges (see, for example, Walters and Webster [8]). However, in the present work, we shall confine attention to the dynamics of the flow, which is often studied through the so-called Couette correction $\mathrm{C}$, defined by

$$
C=\left[\Delta p-\Delta p_{u} L_{u}-\Delta p_{d} L_{d}\right] / 2 \sigma_{\mathrm{w}}
$$

Here, $\Delta p$ is the total pressure difference between the inlet and outlet transducers, $\Delta p_{u}$ is the fully-developed pressure gradient in the upstream section, $\Delta p_{d}$ is the fully-developed pressure gradient in the downstream section, $L_{u}$ and $L_{d}$ are their respective lengths, $\sigma_{\mathrm{w}}$ is the fully-developed wall shear stress in the downstream channel.

The Couette correction $\mathrm{C}$ is usually plotted as a function of the Deborah number

$$
\mathrm{De}=\lambda \dot{\gamma}_{\mathrm{w}}
$$

$\lambda$ is a characteristic relaxation time and $\dot{\gamma}_{\mathrm{w}}$ is the shear-rate at the downstream wall.

In this paper, the Deborah number is defined as $\mathrm{De}=$ $\lambda \dot{\gamma}_{\text {avg }}$, where $\dot{\gamma}_{\text {avg }}$ is the average shear-rate in the constriction zone.

An alternative measure of 'resistance to flow' is the so-called Excess Pressure Drop (epd), defined by Binding et al. [20].

$$
p^{*}=\frac{\left(\Delta p-\Delta p_{f d}\right)_{\mathrm{B}}}{\left(\Delta p-\Delta p_{f d}\right)_{\mathrm{N}}}, \Delta p_{f d}=\Delta p_{u} L_{u}+\Delta p_{d} L_{d}
$$

In this form, $p^{*}$ can be equated to the ratio of Couette corrections for constant-viscosity Boger and Newtonian fluids, with corresponding wall shear stress.

The subscripts $\mathrm{N}$ and $\mathrm{B}$ represent the corresponding Newtonian and Boger fluid values, respectively, when these are applicable.

In our recent computational work on contraction flows (Walters et al. [11-13,21]), we decided to concentrate on the related contraction-expansion $(4: 1: 4)$ geometry, with rounded corners (see Figure 1).

We did this for a number of different reasons. For example:

1) We found the geometry to be far easier to handle in the computations than the conventional 4:1 geometry with sharp corners. Pressure differences were an order of magnitude lower for the 4:1:4 geometry than with 4:1 geometry flows, with shorter downstream distances demanded to establish relaxed stress beyond the constriction (see also Szabo et al. [15] for FENE results). We have certainly been able to reach higher values of the Deborah number in the simulations. Furthermore, application of the basic numerical method was already well developed in the Swansea research group.

2) Importantly, experimental data for the $4: 1: 4$ geometry had been supplied by McKinley and his coworkers (see, for example, Rothstein and McKinley

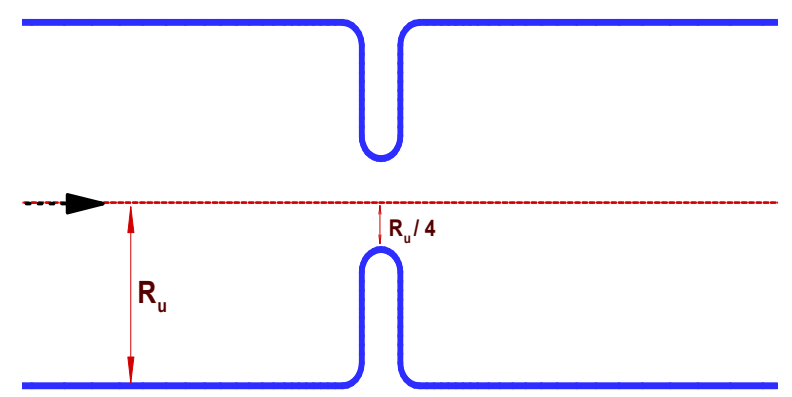

Figure 1. Schematic diagram of contraction-expansion geometry. 
[5]). These showed the same trends as those already well known in the conventional 4:1 geometry. Of major importance was the appearance in the Rothstein and McKinley experiments of substantial increases in the epd for increasing Deborah numbers in the case of Boger fluids.

\section{CONSTITUTIVE EQUATIONS}

We now need to address the question of choosing appropriate constitutive equations for the Boger fluids, which we shall concentrate on in this publication.

Confining attention to incompressible fluids, we can write the Cauchy stress tensor $\sigma_{i k}$ in the form:

$$
\sigma_{i k}=-p \delta_{i k}+T_{i k}
$$

where $p$ is an arbitrary isotropic pressure, $\delta_{i k}$ the Kronecker delta, and $T_{i k}$ is the so-called extra-stress tensor.

Constitutive equations relate the extra-stress tensor $T_{i k}$ to a suitable kinematic variable such as the rateof-strain tensor $d_{i k}$. For two specific but very different reasons, it is often convenient to introduce a so- called "stress splitting":

$$
T_{i k}=T_{i k}^{(1)}+T_{i k}^{(2)}
$$

and to write $T_{i k}^{(1)}$ as a Newtonian contribution

$$
T_{i k}^{(1)}=2 \eta_{1} d_{i k}
$$

Computational rheologists have often found that the introduction of the Newtonian component can greatly assist in the numerical simulation of complex flows, and experimental rheologists, particularly those working with Boger fluids, have also found the modification to be useful. They invariably associate $\eta_{1}$ with the solvent viscosity.

As an example of this stress splitting, consider the well-known Oldroyd B model, with constitutive equations are usually expressed in the form:

$$
T_{i k}+\lambda_{1} \stackrel{\nabla}{T}_{i k}=2 \eta_{0}\left[d_{i k}+\lambda_{2} \stackrel{\nabla}{d_{i k}}\right]
$$

where the triangle denotes the usual upper-convected time derivative introduced by Oldroyd [22].

It is often convenient to write this equation in the form:

$$
\begin{gathered}
T_{i k}^{(1)}=2 \eta_{0} \beta d_{i k} \\
T_{i k}^{(2)}+\lambda_{1} T_{i k}^{(2)}=2 \eta_{0}(1-\beta) d_{i k}
\end{gathered}
$$

where $\beta=\lambda_{1} / \lambda_{2}$.

For the Boger fluids, which have been used in many fundamental experimental contraction-flow studies (see, for example, Boger and Walters [23]), the polymer con- tribution to the total viscosity is very low. This is dominated by the solvent contribution, so that $\beta$ is usually in the range 0.9 to 0.95 , or even higher.

The important rheometrical functions for the Oldroyd $\mathrm{B}$ model are given by

$$
\begin{aligned}
& \eta=\eta_{0}, \\
& \mathrm{~N}_{1}=2 \eta_{0}(1-\beta) \lambda_{1} \dot{\gamma}^{2}, \quad \mathrm{~N}_{2}=0, \\
& \eta_{E}=3 \beta \eta_{0}+3(1-\beta) \eta_{0}\left[\frac{1}{1-\lambda_{1} \dot{\varepsilon}-2 \lambda_{1}^{2} \dot{\varepsilon}^{2}}\right]
\end{aligned}
$$

We see that the Oldroyd B model predicts a constant shear viscosity $\eta_{0}$, a quadratic first normal stress difference $\mathrm{N}_{1}$, a zero second normal stress difference $\mathrm{N}_{2}$, and a potentially high extensional viscosity $\eta_{E}$. In fact, $\eta_{E}$ reaches an infinite value at a finite value of the extensional strain rate $\dot{\varepsilon}$.

As we have indicated, shear thinning is (virtually) absent in Boger fluids. Furthermore, the uniaxial extensional viscosity levels can be very high indeed. These factors have been the main reasons for the popularity of the Oldroyd B model in Computational Rheology studies for Boger fluids. The relative simplicity of the model has been another factor of importance.

However, at this point, it needs to be stressed again that all existing simulations for the Oldroyd B have been unable to predict the large increases in the Couette correction found when Boger fluids flow through axisymmetric contraction/expansion flows (see, for example Walters et al. [11-13]).

With this in mind, Walters et al. [12,13] introduced some new constitutive models to help elucidate the situation. These were in part guided by ideas put forward by Debbaut and Crochet (see Debbaut and Crochet [1] and Debbaut et al. [2]). In these papers, use was made of two rate-of-strain invariants, which we shall conveniently refer to as $\dot{\gamma}$ and $\dot{\varepsilon}$ in what follows:

$$
\dot{\gamma}=2 \sqrt{\mathbf{I I}_{d}}, \quad \dot{\varepsilon}=3 \mathbf{I I I}_{d} / \mathbf{I I}_{d}
$$

Here, $\mathbf{I I}_{d}$ and $\mathbf{I I I}_{d}$ are the two nonzero invariants of the rate of strain tensor $d_{i k}$ in their usual form:

$$
\mathbf{I I}_{d}=\frac{1}{2} \operatorname{tr}\left(\boldsymbol{d}^{2}\right), \quad \mathbf{I I I}_{d}=\operatorname{det}(\boldsymbol{d})
$$

The reason for the choice (14) instead of (15) has been fully explained by Debbaut and Crochet [1] and Debbaut et al. [2]. Clearly, the invariant $\dot{\gamma}$ reduces to the usual shear rate in a steady simple shear flow and the invariant $\dot{\varepsilon}$ reduces to the usual extensional strain rate in a uniaxial extensional flow. Hence, the reason for the notation, Walters et al. [12,13] introduced four constitutive models that they defined as A-D, all of which have the structure introduced in Eqs.8 to 10. 
Model A is simply the Newtonian fluid with $T_{i k}^{(2)}$ given by

$$
T_{i k}^{(2)}=2 \eta_{0}(1-\beta) d_{i k}
$$

Model D is the Oldroyd B model we have already introduced in Eqs.11 and 12 with rheometrical functions given in Eq.13.

Models B and C can be seen as extensions of the models GNM1 and UCM1 in the Debbaut et al. [2] papers. B is an inelastic model with $T_{i k}^{(1)}$ given as in Eq.12 and

$$
T_{i k}^{(2)}=\frac{2 \eta_{0}(1-\beta) d_{i k}}{\left(1-\lambda_{1} \dot{\varepsilon}-2\left(\lambda_{1} \dot{\varepsilon}\right)^{2}\right)}
$$

The rheometrical functions for model $\mathrm{B}$ are

$$
\begin{aligned}
& \eta=\eta_{0}, \\
& \mathrm{~N}_{1}=0, \\
& \eta_{E}=3 \beta \eta_{0}+3(1-\beta) \eta_{0}\left[\frac{1}{1-\lambda_{1} \dot{\varepsilon}-2 \lambda_{1}^{2} \dot{\varepsilon}^{2}}\right]
\end{aligned}
$$

i. e. the same $\eta$ and $\eta_{E}$ as the Oldroyd B model, but with $\mathrm{N}_{1}=0$.

Model C has viscoelastic properties with $T_{i k}^{(1)}$ given as in Eq.12 and

$$
\begin{aligned}
& T_{i k}^{(2)}+\lambda_{1} T_{i k}^{(2)}=2 \eta(\dot{\gamma}, \dot{\varepsilon}) d_{i k}, \\
& \eta(\dot{\gamma}, \dot{\varepsilon})=\eta_{0}(1-\beta)\left(1-\lambda_{1} \dot{\varepsilon}-2\left(\lambda_{1} \dot{\varepsilon}\right)^{2}\right)
\end{aligned}
$$

In this case, the rheometrical functions are

$$
\begin{aligned}
& \eta=\eta_{0}, \\
& \mathrm{~N}_{1}=2 \eta_{0}(1-\beta) \lambda_{1} \dot{\gamma}^{2}, \quad \mathrm{~N}_{2}=0, \\
& \eta_{E}=3 \eta_{0} .
\end{aligned}
$$

This time it is $\eta$ and $\mathrm{N}_{1}$ that match the expressions for the Oldroyd B model, but $\eta_{E}$ now has the Newtonian expression.

Model C can be viewed as a "Generalized WhiteMetzner model" (see, for example Walters et al. [12]).

The benefit of having the four A-D models available was that it allowed us the luxury of the following comparisons:

1) A comparison of the simulations for models $A$ and $\mathrm{B}$ provided an indication of the effect of extensional viscosity on flow characteristics, since $\eta=\eta_{0}$ and $\mathrm{N}_{1}=$ 0 for both models.

2) A comparison of the simulations for models $A$ and $\mathrm{C}$ provided an indication of the effect of "normal stresses" on flow characteristics, with a "Newtonian" extensional viscosity in both.

3) A comparison of the simulations for models B and $\mathrm{C}$ provided an indication of the relative strengths of normal stress and extensional viscosity effects on flow characteristics.

4) A comparison of the simulations for models $C$ and $D$ (i.e. the Oldroyd B model) highlighted further the effect of a high extensional viscosity in the case of elastic liquids.

5) Lastly, a comparison of the simulations for models $\mathrm{B}$ and D highlighted further the normal stress effect, keeping in mind however that, in this comparison, model $\mathrm{B}$ is inelastic and model $\mathrm{D}$ is viscoelastic.

We felt that numerical simulations for the four constitutive models (A-D) could throw considerable light on the influences of the various rheometrical functions on flow characteristics.

We show in Figure 2 simulations for the contraction/expansion geometry provided by Walters et al. $[12,13]$. We summarize their findings here, as these have been fundamental and serve as a basis for the present study, where a number of additional factors are taken into account.

The respective simulations of Figure 2 for the four constitutive models (A-D) demonstrate the influence of the various rheometrical functions on excess pressure drop $(e p d)$ read against increasing deformation rate (De). A comparison of models A to B shows the increasing effect that extensional-viscosity alone has on epd, as both models support vanishing $\mathrm{N}_{1}$. Alternatively, under constant extensional viscosity, a comparison of models A to $\mathrm{C}$ indicates that the increasing influence of normal stress difference can give rise to the opposite effect, that is a decrease in epd (as suggested in Binding [3]).

\subsection{Comparison of Results for Models B and D}

Taking this comparison one step further we may contrast the epd results for model $\mathrm{B}$ with those for model $\mathrm{D}$ (Oldroyd B). Here, we note that the Oldroyd B model reflects the same extensional viscosity as model B (an example of extreme strain-hardening), but with a non-zero normal stress difference of quadratic variation in shear rate (so, $\boldsymbol{N}_{\mathbf{1}} \neq 0$ ). Model D is often used to approximate experimental results for Boger fluids, due to its constant shear viscosity and strain-hardening properties. Consistent with the above, the results again demonstrate a decline in epd from model $\mathrm{B}$ to model $\mathrm{D}$, this being associated with the associated rise in $\boldsymbol{N}_{1}$. We note in addition, that there is the usual upper limit on De attainable in the simulations for model D (attributed to the unbounded nature of $\eta_{e}$ ). Here, there is a slight dip in epd before reaching the limiting value at the Newtonian reference line (for this level of solvent fraction, 0.9), which lies disappointingly short of the large positive epd experimental expectations reported for Boger fluids (Nigen and Walters [4]; Rothstein and McKinley [5]). 


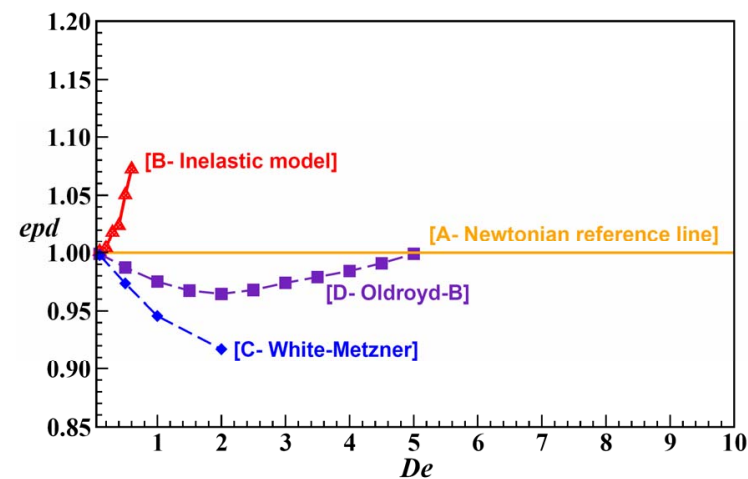

Figure 2. Normalized pressure drop (epd) vs. $\operatorname{De}\left(=\lambda \dot{\gamma}_{\text {avg }}\right)$ for models A-D (cf. Walters et al. [12,13]), $\beta=0.9$.

\subsection{Comparison of Results for Models C and D}

Following this line of study, a direct comparison of epd results for models $\mathrm{C}$ and $\mathrm{D}$, both of which share the same quadratic $\boldsymbol{N}_{\mathbf{1}}$ behavior, represents the effects of extensional viscosity alone (recall that model $\mathrm{C}$ bears a constant extensional viscosity). Here, once again, we discern an increase in epd from model $\mathrm{C}$ to that for model $\mathrm{D}$.

Clearly, the simulations for models A-D contained in Figure 2 provide insights into the possibility of providing numerical simulations which match the experimental data, at least in a semi-quantitative sense. However, we still lack definitive evidence that simulations for a (respectable) constitutive equation, which would have general acceptance, can provide the required agreement between experiment and theory. Hence the reason and motivation for the present work.

We require a model that leads to a constant shear viscosity and possesses other rheometrical features of relevance to Boger-type fluids (also, vanishing $\mathrm{N}_{2}$, see Chilcott and Rallison [16]). For this reason, we have alighted on the so-called FENE-CR (Finite Extendible Nonlinear Elasticity-Chilcott and Rallison [16]) model, which we shall conveniently refer to as model E. This has the following constitutive equations:

$$
\begin{gathered}
T_{i k}=T_{i k}^{(1)}+T_{i k}^{(2)} \\
T_{i k}^{(1)}=2 \eta_{1} d_{i k} \\
T_{i k}^{(2)}=\frac{(1-\beta) f(\operatorname{Tr}(A))(A-I)}{\lambda_{1}}
\end{gathered}
$$

where the stress is expressed through a conformation transformation (A) as

$$
f(\operatorname{Tr}(A)) A+\lambda_{1} \stackrel{\nabla}{A}+f(\operatorname{Tr}(A)) I=0
$$

The stretch function $f(\operatorname{Tr}(A))$ depends on $L$ (the so-called extensibility parameter), and is given by:

$$
f(\operatorname{Tr}(A))=\frac{1}{1-\operatorname{Tr}(A) / L^{2}}
$$

In this equation, $\operatorname{Tr}(A)$ is the trace operator and $L$ essentially measures the size of the polymer molecule in relation to its equilibrium size. $I$ is the identity tensor.

The associated rheometrical functions are given by

$$
\begin{aligned}
& \eta=\eta_{0} \\
& \mathrm{~N}_{1}=\frac{2 \eta_{0}(1-\beta) \lambda_{1} \dot{\gamma}^{2}}{f^{2}}, \quad \mathrm{~N}_{2}=0 \\
& \eta_{e}=3 \beta \eta_{0}+3(1-\beta) \eta_{0}\left[\frac{f}{f^{2}-f \lambda_{1} \dot{\varepsilon}-2 \lambda_{1}^{2} \dot{\varepsilon}^{2}}\right]
\end{aligned}
$$

where $f=f(\operatorname{Tr}(A))$ is defined in Eq.23.

The rheometrical response of the FENE-CR model is displayed in Figures 3 and 4. The model predicts a constant shear viscosity, but the first normal stress difference $\left(\mathrm{N}_{1}\right)$ is weaker than the strong quadratic form exhibited by the Oldroyd-B model. One notes, however, that predictions for large values of the extensibility parameter $(\mathrm{L}>100)$ asymptote to Oldroyd-B behavior in $\mathrm{N}_{1}$ and $\eta_{\mathrm{e}}$. For small values of $\mathrm{L}$ (e.g. $\mathrm{L}=3$ ), significant departure in $\mathrm{N}_{1}$ is observed from an Oldroyd-B response. A monotonic decline in $\mathrm{N}_{1}$ is apparent with decreasing $\mathrm{L}$ (Figure 3).

The extensional viscosity behavior of the FENE- CR model for low extensional strain-rates up to 0.5 is practically identical to that for the Oldroyd-B model. Beyond this station, the extensional viscosity for the FENE-CR model is capped, with the limiting level of the extensional viscosity plateau depending on the elevation of $\mathrm{L}$. This is illustrated in Figure 4, where the trend in extensional viscosity for the FENE-CR model is almost flat for $\mathrm{L}=3$. There is advancing earlier departure from Oldroyd-B trends with appropriate choices of L.

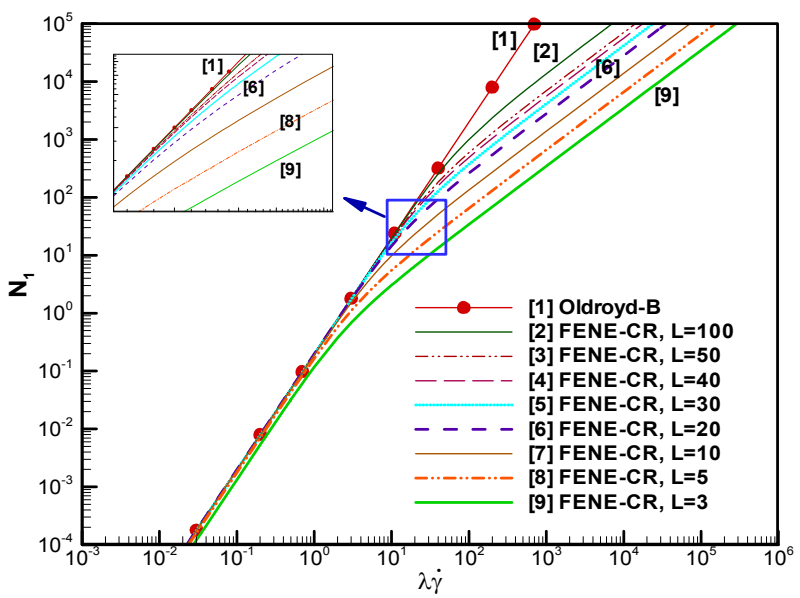

Figure 3. Normal stress data for model E (FENE-CR), increasing $\mathrm{L}$. 


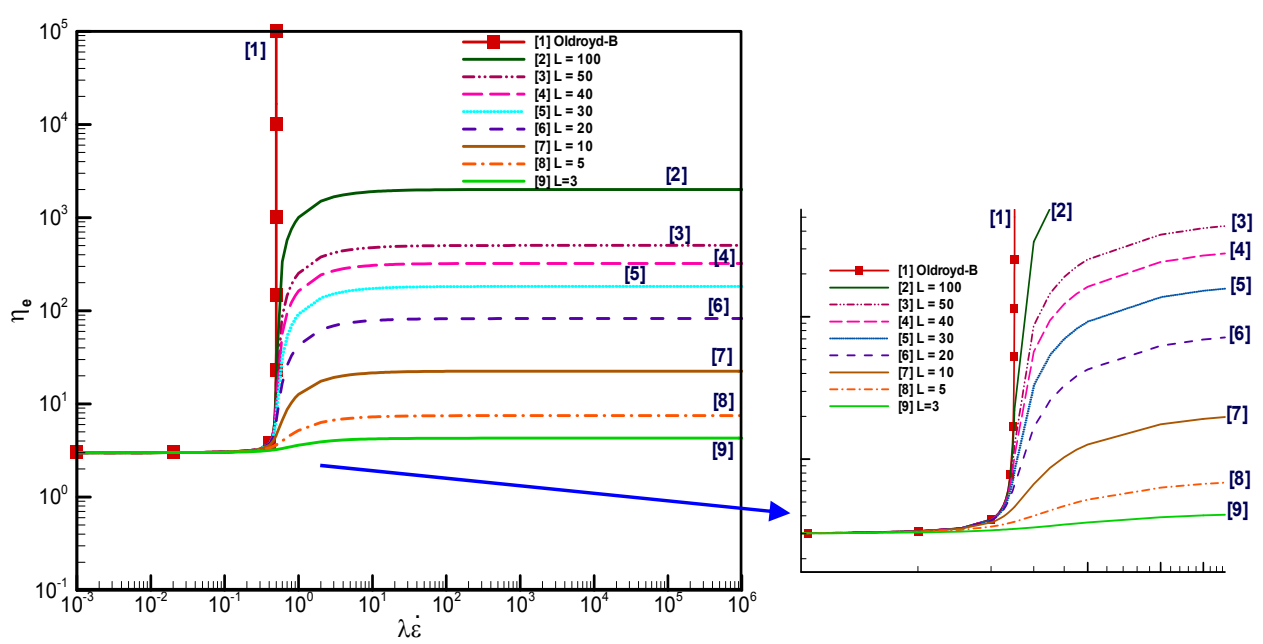

Figure 4. Extensional viscosity data for model E (FENE-CR), increasing L.

These are some of the reasons why we thought that a numerical study of model $\mathrm{E}$ in contraction/expansion flow would be useful.

\section{NUMERICAL BACKGROUND}

\subsection{Hybrid Finite Element/Finite Volume Scheme}

The hybrid finite element/volume scheme employed is a semi-implicit, time-splitting, fractional staged formulation, which draws upon finite element discretization for velocity-pressure approximation and finite volume for stress (see Webster et al. [24], Matallah et al. [25]). Un- der the fe construction, a two-step Lax-Wendroff scheme is employed, a Taylor-Galerkin variant (Donea [26], Zienkiewicz [27]), alongside an incremental pressurecorrection procedure (with $0 \leq \theta_{1} \leq 1$ ). For solenoidal conditions and with a forward time increment factor $\theta_{2}=0.5$, this pressure-correction scheme attracts second-order temporal accuracy, with its incremental form $\left(\theta_{1}>0\right)$ proving superior in uniform temporal error bound attainment over its non-incremental counterpart $\left(\theta_{1}=0\right)$. The three-stage structure can be conveniently expressed (Wapperom and Webster [28]) in semi-discrete representation on the single time step $\left[\mathrm{t}^{\mathrm{n}} ; \mathrm{t}^{\mathrm{n}+1}\right]$, with starting values $\left[\mathrm{u}^{\mathrm{n}} ; \tau^{\mathrm{n}}, \mathrm{p}^{\mathrm{n}}, \mathrm{p}^{\mathrm{n}-\mathrm{1}}\right]$, via:

$$
\begin{aligned}
& \text { Stage 1a: } \frac{2 \operatorname{Re}}{\Delta t}\left(u^{n+\frac{1}{2}}-u^{n}\right)=[\nabla \tau]^{n}+\nabla \cdot\left(2 \beta \frac{d^{n+\frac{1}{2}}+d^{n}}{2}\right)-\nabla\left(p^{n}+\theta_{1}\left(p^{n}-p^{n-1}\right)\right)+F_{G}^{n} \\
& \frac{2 W e}{\Delta t}\left(\tau^{n+\frac{1}{2}}-\tau^{n}\right)=\left(f I-f \tau-\lambda_{1}\left(u \cdot \nabla \tau-L \cdot \tau+\tau \cdot L^{T}\right)\right)^{n} \\
& \text { Stage } 1 b: \quad \frac{\operatorname{Re}}{\Delta t}\left(u^{*}-u^{n}\right)=[\nabla \tau]^{n+(1 / 2)}+\nabla \cdot\left(2 \beta \frac{d^{*}+d^{n}}{2}\right)-\nabla\left(p^{n}+\theta_{1}\left(p^{n}-p^{n-1}\right)\right)+F_{G}^{n+(1 / 2)} \\
& \frac{W e}{\Delta t}\left(\tau^{n+1}-\tau^{n}\right)=\left(f I-f \tau-\lambda_{1}\left(u \cdot \nabla \tau-L \cdot \tau+\tau \cdot L^{T}\right)\right)^{n+\frac{1}{2}}
\end{aligned}
$$

Stage 2: $\quad \nabla^{2}\left(p^{n+1}-p^{n}\right)=\frac{\operatorname{Re}}{\theta_{2} \Delta t} \nabla \cdot\left(u^{*}\right)$

Stage 3: $\frac{2 \operatorname{Re}}{\Delta t}\left(u^{n+1}-u^{*}\right)=-\theta_{2} \nabla\left(p^{n+1}-p^{n}\right)$

Here, $L=\nabla \mathbf{u}$, and the Reynolds number is defined according to convention as $\operatorname{Re}=\rho U \ell / \eta_{0}$, where $\rho$ is the fluid density and $\mathrm{U}, \ell$ are characteristic velocity and length scale of the flow, and $\eta_{0}$ is the total viscosity.
Galerkin discretization may be applied to the embedded Stokesian system components; the momentum equation at Stage 1, the pressure-correction equation at Stage 2 and the incompressibility satisfaction constraint at Stage 3 (to ensure higher order precision). An 
element-y-element Jacobi solution procedure is utilized at Stage 1 (momentum) and Stage 3 for the resulting Galerkin-type Mass matrix-vector equations. This is an efficient and accuracy technique, requiring only a handful of iterations and a mixture of exact and numerical integration rules. This offers a space-time trade-off, so that huge problems may be accommodated (3D, multiple relaxation times, multi-scale). At Stage 2, a direct Choleski decomposition is required, necessitating only a single matrix reduction phase necessary at the outset. Then, semi-implicitness is introduced at Stages 1a,b on pressure and diffusive terms to enhance stability in the strongly viscous regime. Note that, pressure temporal increments invoke multistep reference across three successive time levels $\left[\mathrm{t}^{\mathrm{n}-1}\right.$, $\left.\mathrm{t}^{\mathrm{n}}, \mathrm{t}^{\mathrm{n}+1}\right]$.

\subsection{Finite Volume Fluctuation Distribution Scheme}

The theory may be exposed by first expressing the extrastress equation in non-conservative form, with flux $(\boldsymbol{R}=\mathbf{u} . \nabla \tau$,$) and absorbing remaining terms under the$ source $(\boldsymbol{Q})$, one may obtain:

$$
\frac{\partial \tau}{\partial t}+\boldsymbol{R}=\boldsymbol{Q}
$$

Then, cell-vertex $f v$-schemes are applied to this equation utilizing fluctuation distribution as the upwinding technique, to distribute control volume residuals and furnish nodal solution updates (Wapperom and Webster [29]). We consider each scalar stress component, $\tau$, acting on an arbitrary volume $\Omega=\sum_{l} \Omega_{l}$, whose variation is controlled through corresponding components of fluctuation of the flux $(R)$ and the source term $(\boldsymbol{Q})$,

$$
\frac{\partial}{\partial t} \int_{\Omega_{l}} \tau d \Omega=-\int_{\Omega_{l}} R d \Omega+\int_{\Omega_{l}} Q d \Omega
$$

Flux and source variations are evaluated over each finite volume triangle $\left(\Omega_{l}\right)$, and are subsequently allocated by the chosen cell-vertex distribution scheme to its three vertices. In this manner, by summing all contributions from its control volume $\Omega_{l}$, the nodal update is obtained composed of all $f v$-triangles surrounding node $(l)$. The flux and source residuals may be evaluated over two separate control volumes associated with a given node $(l)$ within the $f v$-cell $\mathrm{T}$. This generates a contribution governed over the $f v$-triangle T, $\left(R_{T}, Q_{T}\right)$, and that subtended over the median-dual-cell zone, $\left(R_{m d c}, Q_{m d c}\right)$. For reasons of temporal accuracy, this procedure demands appropriate area-weighting to maintain consistency, with extension to time-terms likewise. A generalized $f v$-nodal up- date equation may be expressed per stress component, by separate treatment of individual time derivative, flux and source terms, and integrating over associated control volumes, yielding,

$$
\begin{aligned}
& {\left[\sum_{\forall T_{l}} \delta_{T} \alpha_{l}^{T} \Omega_{T}+\sum_{\forall M D C_{l}}\left(1-\delta_{T}\right) \hat{\Omega}_{l}^{T}\right] \frac{\Delta \tau_{l}^{n+1}}{\Delta t}} \\
& =\sum_{\forall T_{l}} \delta_{T} \alpha_{l}^{T} b^{T}+\sum_{\forall M D C_{l}}\left(1-\delta_{T}\right) b_{l}^{M D C}
\end{aligned}
$$

where $b^{T}=\left(-R_{T}-Q_{T}\right), \quad b_{l}^{M D C}=\left(-R_{M D C}+Q_{M D C}\right)^{l}$, $\Omega_{T}$ is the area of the $f v$-triangle $T$, and $\hat{\Omega}_{l}^{T}$ is the area of its median-dual-cell (MDC). The weighting parameter, $0 \leq \delta_{T} \leq 1$, directs the balance taken between the contributions from the median-dual-cell and the $f v$ triangle $T$. The discrete stencil of Eq.27 identifies fluctuation distribution and median dual cell contributions, area weighting and upwinding factors $\left(\alpha_{l}^{T}-\right.$ scheme dependent). The interconnectivity of the $f v$-triangular cells $\left(T_{i}\right)$ surrounding the sample node $(l)$, the blue-shaded zone of $m d c$, the parent triangular $f e$-cell, and the fluctuation distribution ( $f v$-upwinding) parameters $\left(\alpha_{i}^{T}\right)$, for $i=l, j, k$ on each $f v$-cell, are all features illustrated in Figure 5.

\section{NUMERICAL PREDICTIONS}

As we have indicated, the benchmark problem we wish to address is that of flow within a 4:1:4 axisymmetric rounded-corner contractions (Figure 1). In this study, creeping flow is assumed $\left(\mathrm{Re} \approx 10^{-2}\right)$. We concentrate again on the epd defined in Eq.7, rather than the Couette correction, and we restrict attention to $\beta=0.9$ and $1 / 9$.

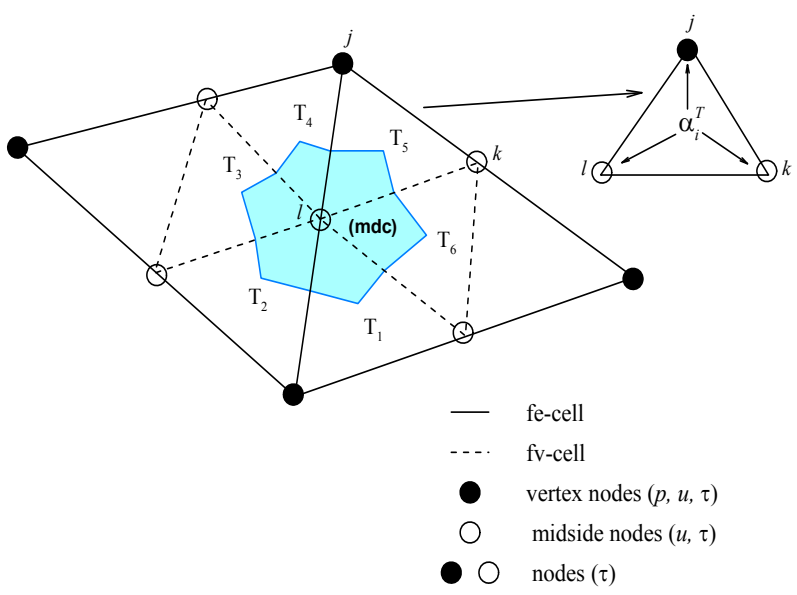

Figure 5. Spatial discretisation: fe-cell with four fv-subcells and fv control volume for node $l$ with median-dual-cell (shaded). 


\subsection{Effect of Normal Stress $\left(\mathrm{N}_{1}\right)$ and Extensional Viscosity $\left(\eta_{\mathrm{e}}\right)$ on epd Comparison of Results for Models D (Oldroyd B) and E (FENE-CR)}

The important new simulation results are contained in Figures 6-10, in which we see epd data for both the Oldroyd B model and variants of the FENE-CR model.

It is clear that our simulations for the Oldroyd B model reach a limit at $D e_{\text {crit }}=5.1$ (see Figure 8), evidently due to unbounded $\eta_{\mathrm{e}}$ and over-strong $\mathrm{N}_{1}$; and we have been unable to reach higher Deborah numbers. Hence, unfortunately, for $\beta=0.9$, we have been unable to break through the epd=1.0 barrier ( $e p d=0.999792$ at $D e$ $=5.1$, whilst for $\beta=0.95$, a positive value of epd is obtained. We have previously confirmed such results through spatial resolution with three different meshes; see Aguayo et al. [10].

This state of affairs may be contrasted with that for the FENE-CR model, which is bounded in extension and has a weakened $\mathrm{N}_{1}$ behavior (see Figure 3). As above, we can anticipate a lowering of epd values due to $\eta_{\mathrm{e}}$ damping and an elevation due to $\mathrm{N}_{1}$ damping. There must clearly be a trade-off between these two factors; but at least we have been able to reach relatively high values of $D e$ in the FENE-CR simulations. Over the range of $D e$ values covered in Figure 6, we see an increase in epd values for $\mathrm{L}=5$ of approximately $15 \%$ at $D e=10$, and in the extended range covered in Figure 7 this rises to approximately $28 \%$. Here, in the several decades beyond $D e=10, \eta_{\mathrm{e}}$ is noted to approach its limiting upper plateau, so that continued further increase in epd can only be attributed to continual weakening of $\mathrm{N}_{1}$ from its quadratic form. So, from a qualitative standpoint, much progress has been made, although it must be admitted that we are still some way from the extravagant increases in epd found in some of the experiments on Boger fluids.

In Aguayo et al. [10] and for steady flow, it was shown that epd in these 4:1:4 flows can only be influenced by shear and extensional contributions within the geometry constriction region excluding the shear upstream-downstream zones. Below we note just how important the shear component $\left(\mathrm{N}_{1}\right)$ can be in this mixed flow region (best interpreted in a Lagrangian sense). One can attempt to segregate the various competing factors through comparison with pairings of L-parameter epd data. For example, comparing data in Figure 6 for lower values of $\mathrm{L}=\{3,5\}$ and the deformation rate range $0 \leq D e \leq 3.33$, one can observe the elevating effect from $\mathrm{L}=5$ to $\mathrm{L}=3$ on epd of gradually weakening $\mathrm{N}_{1}$ (which is the dominant influence in this range). This outcome cannot be attributed to the declining influence of $\eta_{\mathrm{e}}$ (see Figure 4), as if anything, this would have a counter-effect on epd. Subsequently, in the range $D e>3.33$, extensional viscosity effects begin to dominate, as observed in the consistently larger epd-values for $\mathrm{L}=5$; leading to upper limits at $D e=10$ (above the Newtonian reference level) some three times larger with $\mathrm{L}=5(e p d=1.15)$ than for $\mathrm{L}=3$ $(e p d=1.05)$. Note, that the transition zone-point $(D e \approx$ 3.33) is highlighted in Figure 6, where $\mathrm{N}_{1}$ and $\eta_{\mathrm{e}}$ influential dominance switches over.

The same argument for domination of $\mathrm{N}_{1}$-weakening/ $\eta_{\mathrm{e}}$-strengthening holds true for the $\mathrm{L}=\{3,10\}$ and $\{5,10\}$ data; with shifts in the transition point to $D e \approx 7$ for $\mathrm{L}=\{3,10\}$ and to $D e \approx 8.5$ for $\mathrm{L}=\{5,10\}$. For $\mathrm{L}=10$ data and in contrast to Oldroyd-B epd-data in Figure 8, weakening $\mathrm{N}_{1}$ behavior for the FENE-CR model elevates $e p d$ up to the intersection/transition point $(D e \approx 2.5)$. Over $2.5 \leq D e \leq 5$, the stronger $\eta_{\mathrm{e}}$ of the Oldroyd model increasing prevails, so that larger $\eta_{\mathrm{e}}$ gives larger $e p d$, a trend anticipated to continue as $D e$ rises $\left(n b . D e_{c r i t}=5.1\right.$, Oldroyd-B). In contrast, the $\mathrm{F}$ extension/weakening of $\mathrm{N}_{1}$ ) achieves a $D e_{c r i t}=9$ at which the increase in epd advances to around $18 \%$.

Considering larger values of the extensibility parameter ( $L \geq 40$, with larger $\eta_{\mathrm{e}}$ plateaux) in Figure 9, all FENECR epd results are now qualitatively similar to thosefor the Oldroyd-B model, with transition point (gov-

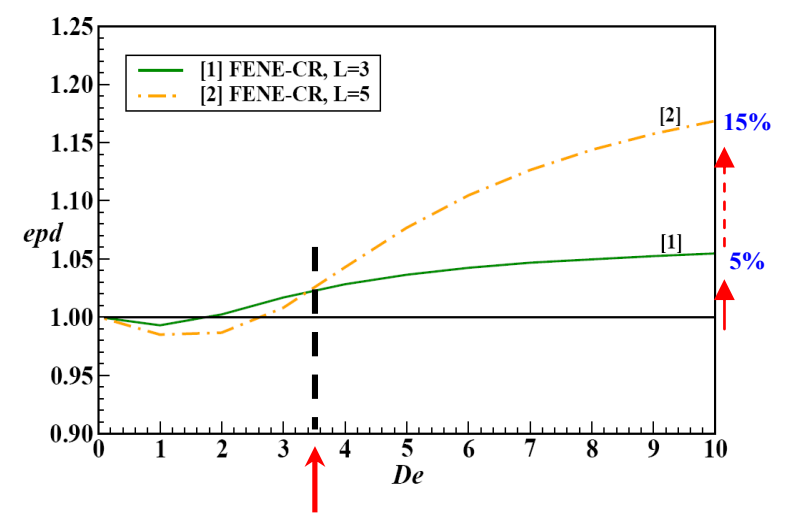

Figure 6. Normalised pressure-drop (epd) vs $D e\left(=\lambda \dot{\gamma}_{\text {avg }}\right)$ for model $\mathrm{E}$ (FENE-CR, $\mathrm{L}=3,5), \beta=0.9$.

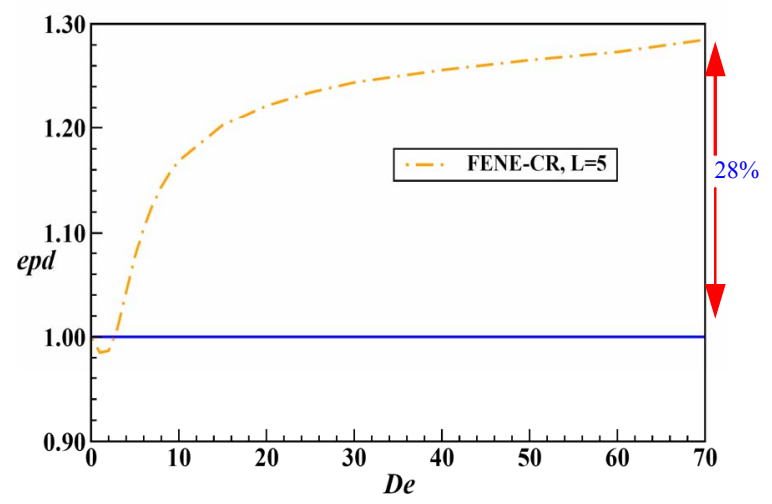

Figure 7. Normalised pressure-drop (epd) vs $D e\left(=\lambda \dot{\gamma}_{\text {avg }}\right)$ for model $\mathrm{E}$ (FENE-CR, $\mathrm{L}=5), \beta=0.9$. 


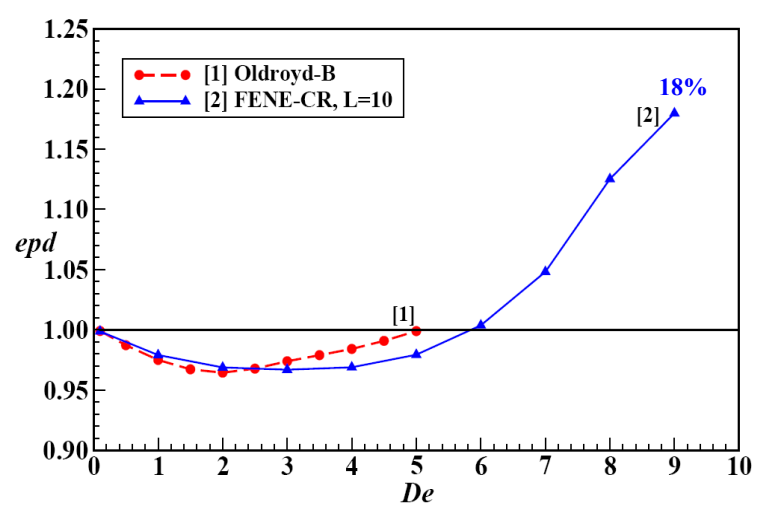

Figure 8. Normalised pressure-drop (epd) vs $D e\left(=\lambda \dot{\gamma}_{\text {avg }}\right)$ for model E (FENE-CR, L=10) and model D (Oldroyd-B), $\beta=0.9$.

erning dominance) shifting to the lower value $D e \approx 2$.

Here, relative to the Oldroyd-B epd-data, there is slight elevation of epd for $D e \leq 2$ ( $\mathrm{N}_{1}$-dominating) and slight suppression of $e p d$ for De $>2$ ( $\eta_{\mathrm{e}}$-dominating).

For completeness, we include simulations for a (lower) value of solvent fraction $\beta$, namely $\beta=1 / 9$. In the early days of Computational Rheology, this value was favored, the reasoning being associated with the realization that, for some models such as the so-called Corotational Oldroyd Model, higher values of $\beta$ would lead to a nonmonotonic shear-stress/shear-rate response. It must be emphasized that such a restriction is not relevant to the Oldroyd B model and is certainly not appropriate for Boger fluids, where we have argued that $\beta=0.9$ (and higher) is more realistic. However, to enable a comparison to be made with some earlier studies, we include in Figure 10 simulations for $\beta=1 / 9$ (low solvent fraction, high polymeric contribution).

These simulations are in general agreement with those of Szabo et al. [15], who also noted epd enhancement for $\mathrm{L}=5, \beta=1 / 9$, accompanied with the explanation that..."a particular value of $\mathrm{L}=5$ needed less stretch to achieve full extension" and a sink flow (pure extension) analysis to identify pressure drop upturn and vortex behavior. We observe, by inspection across L-values at fixed $D e$ for $\beta=1 / 9$, that it is the dominating influence of $N_{1}$ that is clearly apparent with no transition points to $\eta_{\mathrm{e}}$ domination. Interpreted at any particular $D e$, as $\mathrm{L}$ rises, $\mathrm{N}_{1}$ increases in strength and epd declines monotonically. Under such conditions, $\eta_{\mathrm{e}}$-plateaux also rise and so would be anticipated to only contribute to epd-enhancement. Hence, the suppressive influence of $\mathrm{N}_{1}$-rise must counter this to generate decline in epd.

Viewed at each L-value and rising $D e$, earlier arguments reapply governing the competing influences upon epd. This yields a rise of around $13 \%$ in epd for $\mathrm{L}=3$ at $D e=4$. In contrast, the position is adjusted accordingly for $\mathrm{L}=5$ and $\mathrm{L}=10$ data, with gradual strengthening of $\mathrm{N}_{1}$-influence. Hence, for $\mathrm{L}=10$, one detects a local minimum at $D e=3$, beyond which there is an 'upturn'.

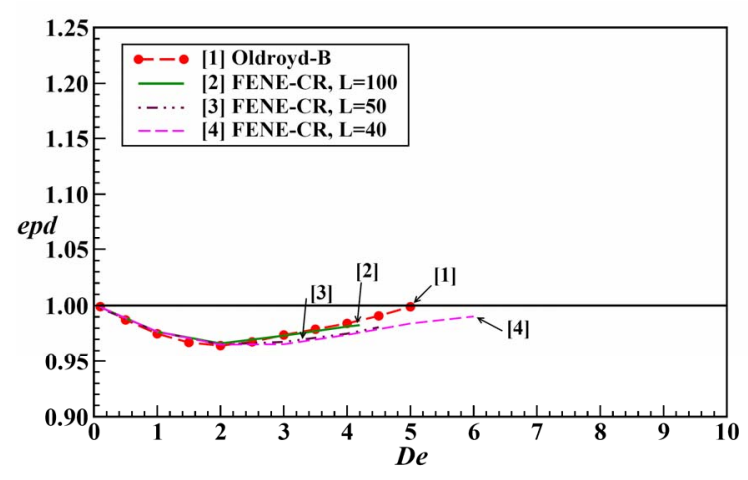

Figure 9. Normalized pressure-drop (epd) vs $D e\left(=\lambda \dot{\gamma}_{\text {avg }}\right)$ for model $\mathrm{E}$ (FENE-CR, $\mathrm{L}=40,50,100)$ and model $\mathrm{D}$ (Oldroyd-B), $\beta=0.9$.

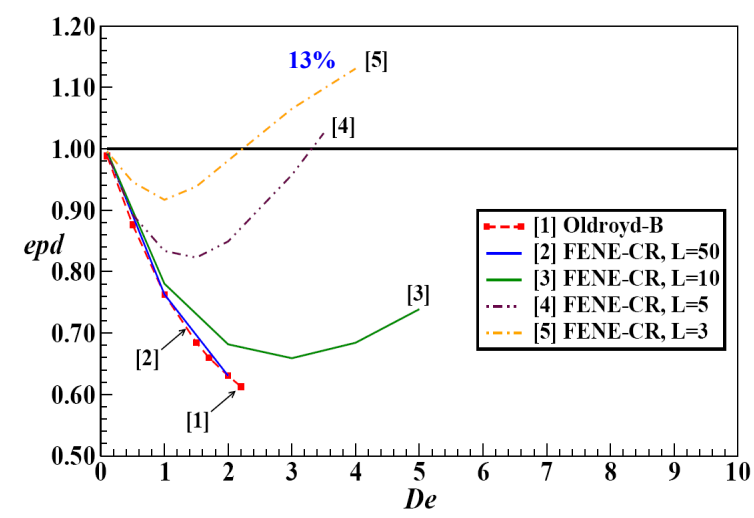

Figure. 10. Normalised pressure-drop (epd) vs $D e\left(=\lambda \dot{\gamma}_{\text {avg }}\right)$ for model E (FENE-CR, L=3-50) and model D (Oldroyd-B), $\beta=1 / 9$.

However, the overall lowering of epd-level at $\mathrm{L}=10$ is such that there is insufficient recovery for $D e>3$ to cross the Newtonian reference line, as occurs with $\mathrm{L}=3$ and $\mathrm{L}=5$ epd-data.

\section{CONCLUSIONS}

The current work has covered the numerical prediction of the excess pressure drop (epd) for the flow of FENECR fluids through a 4:1:4 contraction/expansion geometry with rounded corners. A detailed study of epd and associated parameters has revealed some interesting and provocative results.

Unlike the inadequacies of the Oldroyd B model in observing the experimentally observed increases in epd, various forms of the FENE CR model, possessing first normal stress differences weaker than the strong quadratic form of the Oldroyd B model, are capable of predicting enhanced epd.

We are encouraged by the fact that we have been able to reach epd elevations of nearly $30 \%$, and this is clearly a step in the right direction. However, this is still some way short of some of the large epd-levels found experi- 
mentally for Boger fluids. In addition, we are a little concerned that the various parameters in the FENE-CR model, such as L, are not physically representative at the molecular level, (something which is of course well documented in the literature), and, more importantly, that they have needed to be chosen so precisely in the present work to obtain the desired effect. This suggests some form of dynamic and locally rate-responsive scale would be more appropriate, as commended through the FENEAdaptive- Length-Scale (ALS) model (Ghosh et al. [30]).

So, there are still questions to be answered, but at least some progress has been (and is being) made! In the future, this work will be extended to analyze the additional adjustment when shear-thinning properties are introduced; and also alternative flow scenarios where energy related issues are pertinent, such as drag characteristics in flow past a sphere.

\section{REFERENCES}

[1] Debbaut, B. and Crochet, M.J. (1988) Extensional effects in complex flows. J Non-Newton Fluid Mech, 30, 169-184.

[2] Debbaut, B., Crochet, M.J., Barnes, H.A. and Walters, K. (1988) Extensional effects in inelastic liquids. Xth Inter. Congress on Rheology, Sydney, 291-293.

[3] Binding, D.M. (1991) Further considerations of axisymmetric contraction flows. J Non-Newton Fluid Mech, 41, $27-42$.

[4] Nigen, S. and Walters, K. (2002) Viscoelastic contraction flows: comparison of axisymmetric and planar configurations. J Non-Newton Fluid Mech, 102, 343-359.

[5] Rothstein, J.P. and McKinley, G.H. (2001) The axisymmetric contraction-expansion: The role of extensional rheology on vortex growth dynamics and the enhanced pressure drop. J Non-Newton Fluid Mech, 98, 33-63.

[6] Phillips, T.N. and Williams, A.J. (2002) Comparison of creeping and inertial flow of an Oldroyd B fluid through planar and axisymmetric contractions. J Non-Newton Fluid Mech, 108, 25-47.

[7] Aboubacar, M., Matallah, H., Tamaddon-Jahromi, H.R. and Webster, M.F. (2002) Numerical prediction of extensional flows in contraction geometries: Hybrid finite volume/element method. J Non-Newton Fluid Mech, 104, $125-164$

[8] Walters, K. and Webster, M.F. (2003) The distinctive CFD challenges of computational rheology. Inter $J$ for Numer Meth in Fluids, 43, 577-596.

[9] Alves, M.A., Oliveira, P.J. and Pinho, F.T. (2004) On the effect of contraction ratio in viscoelastic flow through abrupt contractions. J Non-Newton Fluid Mech, 122, 117-130.

[10] Aguayo, J.P., Tamaddon-Jahromi, H.R. and Webster, M.F. (2008) Excess pressure-drop estimation in contraction flows for strain-hardening fluids. J Non-Newton Fluid Mech, 153, 186-205.

[11] Walters, K., Webster, M.F. and Tamaddon-Jahromi, H.R. (2009a) The numerical simulation of some contraction flows of highly elastic liquids and their impact on the relevance of the Couette correction in extensional rheology. Chem Eng Sci, 64, 4632-4639.
[12] Walters, K., Webster, M.F. and Tamaddon-Jahromi, H.R. (2009b) The White-Metzner model then and now. Proceedings of the $25^{\text {th }}$ Annual Meeting of the PPS meeting, Goa, India, 02, 1-14.

[13] Walters, K., Tamaddon-Jahromi, H.R., Webster, M.F., Tomé, M.F. and McKee, S. (2010) The competing roles of extensional viscosity and normal stress differences in complex flows of elastic liquids. Korean-Australian Journal (to be published), 2010.

[14] White, J.L. and Metzner, A.B. (1963) Development of constitutive equations for polymeric melts and solutions. J Appl Polym Sci, 7, 1867-1889.

[15] Szabo, P., Rallison, J.M. and Hinch, E.J. (1997) Start-up of flow of a FENE-fluid through 4:1:4 constrictions in a tube. J Non-Newton Fluid Mech, 72, 73-86.

[16] Chilcott, M.D. and Rallison, J.M. (1988) Creeping flow of dilute polymer solutions past cylinders and spheres. $J$ Non-Newton Fluid Mech, 29, 381-432.

[17] Barnes, H.A., Hutton, J.F. and Walters, K. (1989) An introduction to rheology. Elsevier, Amsterdam.

[18] Jackson, K.P., Walters, K. and Williams, R.W. (1984) A rheometrical study of Boger fluids. J Non-Newton Fluid Mech, 14, 173-188.

[19] Tanner, R.I. and Walters, K. (1998) Rheology: An historical perspective, Elsevier Science \& Technology, Netherlands.

[20] Binding, D.M., Phillips, P.M. and Phillips, T.N. (2006) Contraction/expansion flows: The pressure drop and related issues. J Non-Newton Fluid Mech, 137, 31-38.

[21] Walters, K., Webster, M.F. and Tamaddon-Jahromi, H.R. (2008) Experimental and computational aspects of some contraction flows of highly elastic liquids and their impact on the relevance of the Couette correction in extensional rheology. Proc. $2^{\text {nd }}$ Southern African Conference on Rheology (SASOR 2), 1-6.

[22] Oldroyd, J. G. (1950) On the formulation of rheological equations of state. Proc. Roy.Soc., A200, 523-541.

[23] Boger, D.V. and Walters, K. (1993) Rheological phenomena in focus, Elsevier Science Publishers.

[24] Webster, M.F., Tamaddon-Jahromi, H.R. and Aboubacar, M. (2005) Time-dependent algorithms for viscoelastic flow: Finite element/volume schemes. Numer Meth Par Diff Equ, 21, 272-296.

[25] Matallah, H., Townsend, P. and Webster, M.F. (1998) Recovery and stress-splitting schemes for viscoelastic flows. J Non-Newton Fluid Mech, 75, 139-166.

[26] Donea, J. (1984) A Taylor-Galerking method for convective transport problems. Int J Numer Methods Eng, 20, 101-119.

[27] Zienkiewicz, O.C., Morgan, K, Peraire, J., Vandati, M. and Löhner R. (1985) Finite elements for compressible gas flow and similar systems. Seventh International Conference on Computational Methods of Applied Science and Engineering.

[28] Wapperom, P. and Webster, M.F. (1999) Simulation for viscoelastic flow by a finite volume/element method. Comput Meth Appl Mech Eng, 180, 281-304.

[29] Wapperom, P. and Webster, M.F. (1998) A second-order hybrid finite-element/volume method for viscoelastic flows. J Non-Newton Fluid Mech, 79, 405-431.

[30] Ghosh, I., Lak, Joo Y., McKinley, G.H., Brown, R.A. and Armstrong, R.C. (2002) A new model for dilute polymer solutions in flows. $J$ Rheol, 46(5), 1057-1089. 